\title{
Identifying Network Topology Using Travelling Wave Reflections of the Test Signal
}

\author{
Roko JERČIĆ, Ivica PAVIĆ, Domagoj PEHARDA
}

\begin{abstract}
State estimation is an important part of control functions and power system management. Correct insight and interpretation of the network topology are a crucial step of any state estimator. In order to improve state estimators accuracy and speed, a new approach of determining network topology is presented. Presented method is based on reflection measurement of the injected test signal from the impedance discontinuities dependent on the topological state at a particular site in the network. Correct interpretation of the measured reflected signal gives possibility to determine the topological state at the reflection site. This article describes the methodology for topological recognition, while algorithm verification is done by using several cases of topology determination on the example of the test network. The results are given in graphical form showing simulated and expected reflection signal. Matching of simulated and expected reflected signal has proved the correctness of the topological recognition algorithm.
\end{abstract}

Keywords: network topology; state estimation; topology processor; travelling wave; wave reflection

\section{INTRODUCTION}

Transients occurring in a high voltage network can be caused by lightning stroke to transmission line or somewhere in its vicinity, fault initiation and switching operations in high voltage networks. Although the theory of travelling waves has been known since the 40's of the last century, the first applications began about twenty years ago. Currently this theory is most commonly used for fault detection, classification and location on transmission lines [1-3]. The progress in signal processing techniques and intelligent recognition systems occurring in recent years has made it possible to apply the theory of travelling waves in real-time monitoring of power system topology and state estimation.

State estimation is a crucial process for real-time power system operation and control. State estimation solution determines power system state, i.e. voltage magnitudes and angles at all buses which is used for all other power system calculations [4]. One of the key steps in state estimator calculation is topology processing which collects statuses of switches and calculates current topology. For classic state estimators, measurements and switching devices statuses are collected using SCADA systems. These data are not synchronous, and there exists a delay of a few seconds between measurement and its use in state estimator calculation. For classic state estimator this delay is not an issue because it assumes steady state of the power system. With appearance of the synchrophasor measurements fast (real-time) estimators are becoming possible. Such measurements have time tags and have latency of few tens of milliseconds allowing for robust linear state estimators (LSE) $[5,6]$ to be able to calculate the state of the system in real time [7]. Synchrophasor measurements of voltage and current have a linear relation to network state, which provides direct (non-iterative) solution of the state estimation problem [8]. Weighted least squares (WLS) estimator is a usual and quite fast choice for solving state estimation problem where there are more measurements than network states. However, WLS estimator is not robust estimator, in WLS estimator even a single bad measurement can result in a significant bias of the solution. This is the reason why WLS estimators are usually applying the largest normalized residual test to detect and remove bad data measurement [4]. Calculation of residual covariance matrix is computationally expensive which is the reason why fast linear state estimators use robust estimators like the least absolute value (LAV) estimator [6]. Linear state estimator has been adopted by various power utilities but it has difficulty providing good estimate results when it encounters a topology error [4]. Under normal conditions (with no change of topology or model) linear state estimator calculation for a system with several thousand nodes can be completed faster than synchrophasor sample rate. Even after a change in topology it quickly catches up to the synchrophasor sample rate. In the case of using Inter-Control Center Communications Protocol (ICCP) for breaker status update, this data is 2-6 $\mathrm{s}$ delayed from the phase measurement unit (PMU) data. During this time interval LSE may have a mismatch between measurement and model, which can create significant solution errors. Moreover, some breaker statuses may be reported incorrectly [9]. When topology changes are undetected or happen during the SCADA data polling, the estimation is likely to fail due to its dependence on the bus admittance matrix. Changes in system topology often create rapid changes in power system states. Other calculations dependent on state estimator are most needed by operators when rapid changes are happening in power system. Therefore, fast estimation of topology for use in real-time estimator is needed.

\section{METHODOLOGY}

Presented topology processor is based on measuring the reflection of injected test signal from network impedance discontinuity. Since the surge impedance seen at the end of the transmission line depends on the topological condition on terminated bus, by measuring the reflected signal and using telegraph Eqs. (1) and (2), with related knowledge about behaviour of travelling waves in power system, it is possible to specify the network topology of adjacent substations viewed from substations of the injected signal (adjacent substations terminate one transmission line).

$$
\begin{aligned}
& \frac{\partial u(x, t)}{\partial x}=-L \frac{\partial i(x, t)}{\partial t}-R i(x, t) \\
& \frac{\partial i(x, t)}{\partial x}=-C \frac{\partial u(x, t)}{\partial t}-G i(x, t)
\end{aligned}
$$


Injection method and the signal measurement method are not processed in this paper but a theoretical elaboration of the problem and the solutions way are given. For the topological processor presented here it is necessary to inject a voltage test signal into a selected bus with defined amplitude and duration, and the same point measures its reflection from other buses (i.e. points of surge impedance discontinuity) in adjacent substations. Measured reflected signal amplitude depends on the magnitude of injected signal, transmission line attenuation and its surge impedance, and surge impedance seen from transmission line ends. Surge impedance of transmission line is calculated according to the expression (3)

$Z_{v}=\sqrt{\frac{R+j \omega L}{G+j \omega C}}$

or $\sqrt{L / C}$ for the lossless line [10].

All of these dependencies are defined by construction of the lines. Common surge impedance seen from the transmission line ends depends on terminated bus topology. From the measured signal and known construction defined dependencies, using below described algorithm, it is possible to uniquely define the topology in all adjacent substations at a given moment. It is noteworthy that the measured signal includes already reflected signal from its bus (bus in which the signal is being injected). While processing measured signal, it is necessary to consider all relevant components of the signal reflectance connected to the bus where the signal is injected and measured.

\subsection{Test Signal Function}

Selection of the proper test signal is based on some constraints. Duration of the injected signal must be chosen in a way that the length of the waves is shorter than the double length of the smallest transmission line in observed network configuration. Observed configuration considers all transmission lines incident with their own bus (bus to which the test signal is injected) and with all buses connected to adjacent buses. Reason for such requirement is a need that the reflected signal should not interfere with its source. Since it must be of finite duration, test signal consequently contains more frequencies. Therefore, the Fourier transform of the selected signal should have expressed peak around the selected frequency value to minimize the impact of network parameters frequency dependence.

Additionally, the test signal must be a symmetrical three-phase signal. Otherwise the existence of zero sequence component of asymmetric signal where there are different parameters (zero sequence parameters), together with different speed rates of test signal wave, can lead to dysfunction of the recognition algorithm and misinterpretation of results.

\subsection{Topology Recognizing Algorithm}

First, a bus in which test signal is injected and reflection is measured will be called home bus. Its adjacent bus, in which topology should be observed, will be called topology bus. It is necessary to determine optimum home bus position for signal injection. In the radial network it is usually supply transformer secondary winding bus. After defining home bus position, it is necessary to determine the longest line incident with the topology bus without taking into account the length of the line between these buses. Travelling time to home bus of the reflected test signal wave from the opposite end of the longest line connected totopology bus determines observed time. In observed time test signal reflection from opposite ends of all lines that are incident with topology bus will be measured. Crucial step is, by using (4), to define travelling times of all reflected waves from single injected test signal wave to home bus including multiple reflections from same buses in observed time.

$t=\frac{l}{v}=\frac{l}{\frac{1}{\sqrt{L C}}}=l \sqrt{L C}$,

where $t$ represents travelling time, $v$ is surge velocity, $l$ is line length, $L$ and $C$ are inductance and capacitance per unit length respectively.

Travelling time stands for the time of reflected signal amplitude occurring at home bus. It is also considered that injected test signal function has defined amplitude maximum ( $\tau$ - amplitude time) and that the reflected signal has a shape like the injected test function which differs in magnitude depending on the reflection coefficient.

If test signal function is defined as $V(t)$, control function has to be modeled as presented in (5).

$V_{c t r l}(t)=\sum_{i=1}^{N} a_{i} V\left(t-t_{i}+\tau\right)$

where $a_{i}$ is measured reflected signal magnitude, $t_{i}$ is reflected signal travelling time. This control function has a form of expected reflected signal with unknown coefficients $a_{i}$ which have to be determined. $a_{i}$ also represents information on reflection coefficients (ratio between injected and measured amplitude with attenuation included) and it could be determined from the measured signal with expression (6).

$V_{c t r l}\left(t_{j}\right)=V_{m a}\left(t_{j}\right) \forall j \leq N$

by including (5) in (6) is obtained:

$\sum_{i=1}^{N} a_{i} V\left(t_{j}-t_{i}+\tau\right)=V_{m a}\left(t_{j}\right) \forall j \leq N$

In the defined system there are $N$ equations with $N$ variables and it can be written in matrix form (8):

$\left[\begin{array}{ccc}A_{11} & \cdots & A_{1 N} \\ \vdots & \ddots & \vdots \\ A_{N 1} & \cdots & A_{N N}\end{array}\right]\left[\begin{array}{c}a_{i} \\ \vdots \\ a_{n}\end{array}\right]=\left[\begin{array}{c}V_{m a}\left(t_{1}\right) \\ \vdots \\ V_{m a}\left(t_{n}\right)\end{array}\right]$

where $A_{j i}=V\left(t_{j}-t_{i}+\tau\right)$. From (8) follows (9)

$[a]=[A]^{-1}\left[V_{m a}\right]$.

By solving this equation system, vector $a_{i}=\left[a_{1} \ldots a_{N}\right]$ is obtained. Vector elements represent magnitudes of the measured reflected test signal at expected times of 
maximum magnitude arrivals to home bus. $a_{1}$ represents a magnitude of reflected signal in topology bus and $a_{N}$ represents a magnitude of reflected signal from opposite end of the longest line incident with topology bus. With $a_{i}$ and additional requirements explained below, it is possible to determine the topology of the observed bus. If the observed bus, for which we want to determine the topology, besides connected lines includes transformers, first it is important to determine whether and how many of them, are turned on. In travelling waves, theory transformers can faithfully be presented by a lump parameter, meaning that reflection exists at one point only (unlike the transmission line that has a propagation time). Wave impedance of the transformer depends on its geometrical parameters and according to [11] is:

$Z_{T}=\sqrt{\frac{L}{C}}=2 N_{t}\left(\frac{w d}{h w+h d}\right) \sqrt{\frac{\mu_{0}}{\varepsilon_{0}}} \sqrt{\frac{\mu_{r}}{\varepsilon_{r}}}$.

$N_{t} / h$ represents number of windings per unit length, $w$ is the distance between the coil and the core and $d$ is distance from tank to transformer winding. In order to detect the switching status of the transformer on the observed bus, several additional steps are required.

It is known that the amplitude of the reflected wave depends on the impedance seen on the bus of which the wave reflected. In case when several transmission lines or transformers are connected at the same bus, their total surge impedance is a parallel circuit of their surge impedances [12]. So, reflection coefficient $R$ on recent bus amounts to:

$R=\frac{\left(Z_{1}\|\ldots\| Z_{n}\right)-Z_{0}}{\left(Z_{1}\|\ldots\| Z_{n}\right)+Z_{0}}$

$Z_{1} \ldots Z_{n}$ are surge impedances of connected elements, $Z_{0}$ is a surge impedance of connection transmission line (in this case, line between home and topology bus). Overall impedance seen from topology bus can be written as:

$Z_{\text {all }}=\left(Z_{1}\|\ldots\| Z_{n}\right)$,

where:

$\frac{1}{Z_{\text {all }}}=\sum_{p=1}^{P} \frac{1}{z_{p}}+\sum_{q=1}^{Q} \frac{1}{z_{q}}$

or

$Z_{\text {all }}=\frac{1}{\sum_{p=1}^{P} \frac{1}{Z_{p}}+\sum_{q=1}^{Q} \frac{1}{Z_{q}}}$.

$P$ is a number of transmission lines, and $Q$ is a number of transformers connected to the topology bus, $Z_{p}$ and $Z_{q}$ is their surge impedances respectively. Expression for the transmission line wave signal attenuation is:

$A^{*}=A e^{-\frac{R}{L} t}$

A is injected signal amplitude, $R$ and $L$ are transmission line resistance and inductance per unit length, and $t$ is wave travelling time [10]. As it is stated before, measured signal is already reflected signal from its own bus (home bus) and the assumption is that the topology of home bus is known. Now it is possible to write an expression for the impedance seen from topology bus using $\mathrm{a}_{1}$ as magnitude of measured reflected signal at home bus and above assumptions.

$Z_{\text {all }}^{*}=Z_{0} \frac{A\left(1+R_{0}\right) e^{-\frac{R}{L} t_{1}}+a_{1}}{A\left(1+R_{0}\right) e^{-\frac{R}{L} t_{1}}-a_{1}}$

$R_{0}$ represents reflection coefficient at home bus. If it is assumed that the surge impedance of lines is similar to each other and differs a lot from the surge impedance of a transformer, expression (13) can be approximated by:

$\frac{1}{z_{\text {all }}}=\frac{p}{z_{p}}+\frac{q}{z_{q}}$

where $Z_{p}$ and $Z_{q}$ are average surge impedances of possibly connected transmission lines and transformers respectively, and $p, q$ are their number. In order to conclude how many transmission lines and transformers are connected to the topology bus, values of $p$ and $q$ in expression (17) have to befound. It was determined that values of $p$ and $q$ must be such that expression (18) gives minimal value,

$\min \left(\frac{1}{z_{\text {all }}}-\frac{1}{z_{\text {all }}^{*}}\right)^{2}$

In other words, which connection combination of lines and transformers at topology bus provides the measured impedance. This determines the number of transmission lines and transformers connected to the bus. By analyzing elements of [a] vector it is possible to determine which one of the transmission lines is connected. It gives all the amplitudes of reflected signals in the observed period. Since the reflections are expected at the end of the transmission line, the existence of significant value in the expected amplitude point implies connection of transmission line. Missfunction can occur if some of the observed lines are terminated with its characteristic impedance. In that case there is no reflection from end of the line despite being connected to the topology bus. However, from (17) and (18) number of connected lines could be obtained, and from [a] vector which of the expected reflection coefficients is missing. A combination of those conditions determines which of the connected transmission line is possibly closed with its characteristic impedance.

\section{RESULTS AND DISCUSSION}

Since field measurements, required for demonstration of presented approach for creating a topological processor, are still lacking, simple model of a radial test network was made. Test network consists of one feeder transformer connected on $\mathrm{M}$ bus (home bus) in which test signal has been injected. On the same bus one transmission line is connected and terminated with $\mathrm{S}$ bus (topology bus) on the end side in remote substation. On the $\mathrm{S}$ bus there are radial connections of four different transmission lines terminated 
with buses named $\mathrm{K}, \mathrm{R}, \mathrm{P}$ and $\mathrm{Z}$, and one power transformer named TR (Fig. 1).

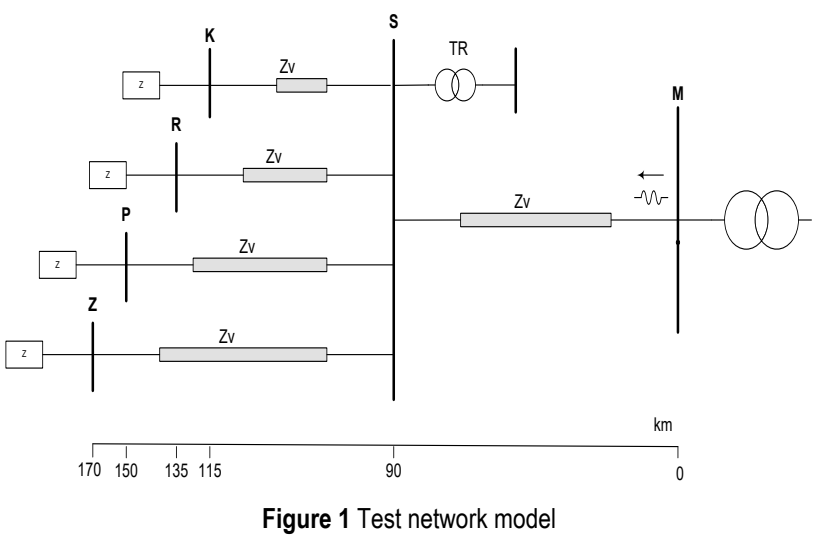

A model of radial test network and simulation of different cases were made in EMTP-RV software, while results were processed using MATLAB. Components of system used in proposed model are modelled as follows and simulation step for each model is $0,5 \mu \mathrm{s}$.

1) Transmission line - three-phase frequency-dependent transmission line model reconstructed using EMTP tool for the reconstruction of frequency-dependent parameters from parameters known at a frequency of $50 \mathrm{~Hz}$. Parameters at a frequency of $50 \mathrm{~Hz}$ are shown in Tab. 1.

2) Transformer - modelled by Ohm resistance obtained by including the structural parameters in the mathematical term (10).

3) Signal generator - modelled as a symmetric threephase AC voltage source of the signal amplitude of $1000 \mathrm{~V}$ at the frequency of $10 \mathrm{kHz}$. In the model only the first half period of injected signal was used.

4) Network equivalent - modeled by Ohm resistance of total amount of the wave impedance $(\Omega)$ in lines connected to the final bus.

Table 1 Line data at $50 \mathrm{~Hz}$

\begin{tabular}{|c|c|c|c|c|c|c|}
\hline & $\begin{array}{c}R \\
(\Omega / \mathrm{km})\end{array}$ & $\begin{array}{c}L \\
(\mathrm{mH} / \mathrm{km})\end{array}$ & $\begin{array}{c}C \\
(\mathrm{nF} / \mathrm{km})\end{array}$ & $\begin{array}{c}R_{0} \\
(\Omega / \mathrm{km})\end{array}$ & $\begin{array}{c}L_{0} \\
(\mathrm{mH} / \mathrm{km})\end{array}$ & $\begin{array}{c}C_{0} \\
(\mathrm{nF} / \mathrm{km})\end{array}$ \\
\hline $\mathrm{M}-\mathrm{S}$ & 0,140 & 1,337 & 8,658 & 0,384 & 4,011 & 5,634 \\
\hline $\mathrm{S}-\mathrm{K}$ & 0,191 & 1,337 & 8,658 & 0,439 & 4,011 & 5,634 \\
\hline $\mathrm{S}-\mathrm{R}$ & 0,192 & 1,337 & 8,594 & 0,442 & 4,011 & 5,793 \\
\hline $\mathrm{S}-\mathrm{P}$ & 0,120 & 1,337 & 8,626 & 0,379 & 3,909 & 5,761 \\
\hline $\mathrm{S}-\mathrm{Z}$ & 0,120 & 1,337 & 8,658 & 0,387 & 3,909 & 5,761 \\
\hline
\end{tabular}

The goal of this simulation is to send test signal from $\mathrm{M}$ bus to $\mathrm{S}$ bus by using signal generator and measuring response to the sending point, by measuring reflected wave. By using method described in previous section, it will be possible to uniquely determine the topology of the bus $\mathrm{S}$ using the reflected wave. In other words, it will be possible to determine which one of the examined transmission lines is connected to the bus.

It is assumed that there are only two (on and off) possible connection states for each transmission line [13], which means that the total number of possible topological states at $\mathrm{S}$ bus equals:

$T=2^{r}$

In proposed network model, together with a transmission line which connects $\mathrm{S}$ and $\mathrm{M}$ buses, there are 5 relevant system components $-r, 4$ transmission lines and one transformer. That with the transmission line M-S in on state gives 32 possible cases. In this paper several specific/selected cases will be presented.

The lengths of connected transmission lines used in this model, its wave impedance and impedance visible from the terminating bus are given in Tab. 2 .

Table 2 Line length and surge impedances

\begin{tabular}{|c|c|c|c|c|c|}
\hline & $\mathrm{M}-\mathrm{S}$ & $\mathrm{S}-\mathrm{K}$ & $\mathrm{S}-\mathrm{R}$ & $\mathrm{S}-\mathrm{P}$ & $\mathrm{S}-\mathrm{Z}$ \\
\hline$l(\mathrm{~km})$ & 90 & 25 & 45 & 60 & 80 \\
\hline$Z_{v}(\Omega)$ & 388,19 & 388,44 & 391,50 & 389,00 & 388,28 \\
\hline$Z_{t}(\Omega)$ & $\left(1500^{*}\right)$ & 0 & 100 & 90 & 95 \\
\hline
\end{tabular}

*Transformer model impedance at $\mathbf{M}$ bus

Calculated amplitude times of the reflected signal in reference time at a given frequency $(10 \mathrm{kHz})$ are shown in Tab. 3. The index associated to the bus tag represents ordinal number of reflections from the same bus measured in the $\mathrm{M}$ bus and double busbar code represents a multiple reflection between two different buses (e.g. RK means transmission of signal through $\mathrm{S}$ to $\mathrm{K}$, reflection from $\mathrm{K}$ and transmission trough $\mathrm{S}$ in other direction to $\mathrm{R}$, reflection from $\mathrm{R}$, transmission through $\mathrm{S}$ again to $\mathrm{M}$ ). Using Tab. 3, vector $[a]$ could be mapped in form

$a_{i}=\left[a_{1} \ldots a_{N}\right]$

$a_{1}$ is amplitude of the reflected signal from $\mathrm{S}$ bus (topology bus), $a_{2}$ is amplitude of reflected signal from $\mathrm{K}$ bus, $a_{3}$ is amplitude of reflected signal from $\mathrm{R}$ bus and so to $a_{8}$ which is amplitude of reflected signal from $\mathrm{Z}$ bus. It is also evident that $a_{4}, a_{6} a_{7}$ are redundant values.

The following results represent six relevant cases. Each case is shown with the simulated response of the reflected signal and modelled control function on the same figure. Also, for each case $[a]$ vector is given in Tab. 4.

Table 3 Reflected signal amplitude times (ms)
\begin{tabular}{|c|c|c|c|c|c|c|c|c|}
\hline & $\mathrm{S}$ & $\mathrm{K}$ & $\mathrm{R}$ & $\mathrm{K} 2$ & $\mathrm{P}$ & $\mathrm{RK}$ & $\mathrm{K} 3$ & $\mathrm{Z}$ \\
\hline$t_{\max }[\mathrm{ms}]$ & 0,62996 & 0,79812 & 0,93276 & 0,96628 & 1,03262 & 1,00914 & 1,13444 & 1,16776 \\
\hline
\end{tabular}

Table $4 a_{i}$ vector of presented cases

\begin{tabular}{|c|c|c|c|c|c|c|c|c|}
\hline & $a_{1}$ & $a_{2}$ & $a_{3}$ & $a_{4}$ & $a_{5}$ & $a_{6}$ & $a_{7}$ & $a_{8}$ \\
\hline Fig. 2 & $-1,0550$ & 0,2223 & $-0,1256$ & $-0,1454$ & $-0,1366$ & $-0,0955$ & 0,0675 & $-0,1202$ \\
\hline Fig. 3 & $-0,6845$ & $-0,0002$ & $-0,3242$ & $-0,0321$ & $-0,3394$ & $-0,0113$ & $-0,0057$ & $-0,0033$ \\
\hline Fig. 4 & $-0,9121$ & $-0,0009$ & $-0,1926$ & $-0,0187$ & $-0,2015$ & $-0,0061$ & $-0,0028$ & $-0,1922$ \\
\hline Fig. 5 & $-0,6876$ & 0,5627 & 0,0052 & $-0,2174$ & $-0,0041$ & 0,0000 & 0,0871 & $-0,3067$ \\
\hline Fig. 6 & $-0,6870$ & 0,5634 & 0,0053 & $-0,2174$ & $-0,3385$ & $-0,0106$ & 0,0814 & 0,0084 \\
\hline Fig. 7 & $-0,2717$ & 1,1338 & 0,0087 & $-0,1713$ & $-0,0025$ & 0,0000 & 0,0279 & 0,0041 \\
\hline
\end{tabular}


Figs. 2-7 represent simulated response of reflected signal measured at $\mathrm{M}$ bus (green line) and the modelled control function (blue line). Time span of presented figures is the same as observable period (travelling time of the reflected test signal wave from the opposite end of the longest line connected to topology bus). In this case it is travelling time from home bus to $\mathrm{Z}$ bus and back. If the values of modelled control functions coincide with the simulated values, especially at amplitude points, then the assumed topology at topology bus is correct. Overlap points of the two functions are those which satisfy the Eq. (3).

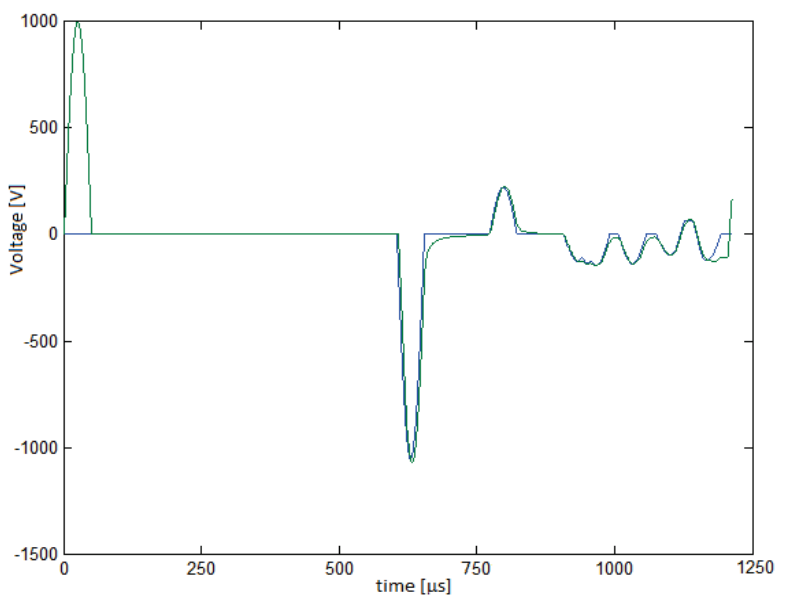

Figure 2 Reflected signal where all lines and TR are connected

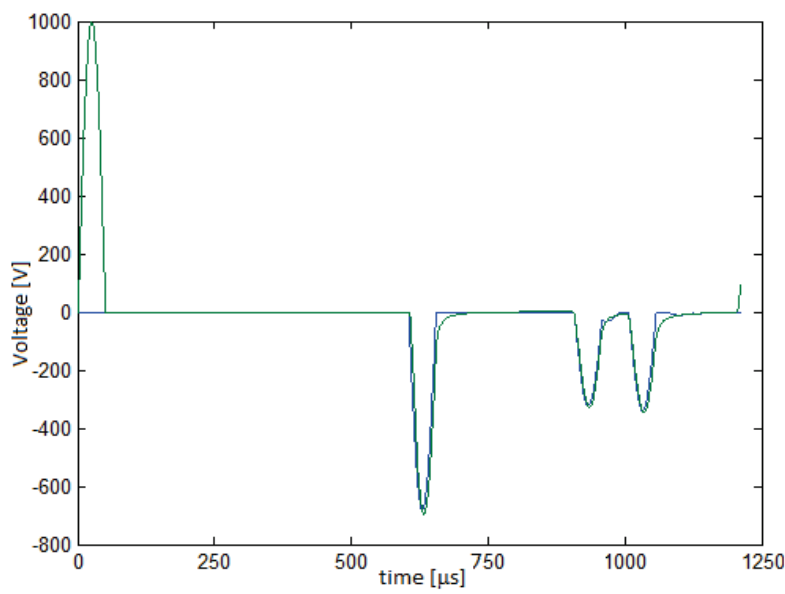

Figure 3 Reflected signal where $\mathrm{K}$ and $\mathrm{Z}$ buses are disconnected

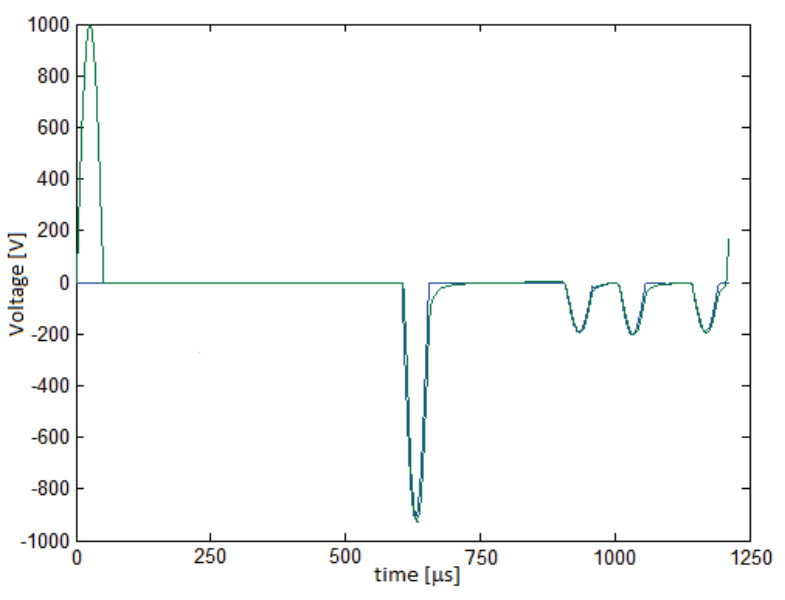

Figure 4 Reflected signal where $\mathrm{K}$ bus is disconnected

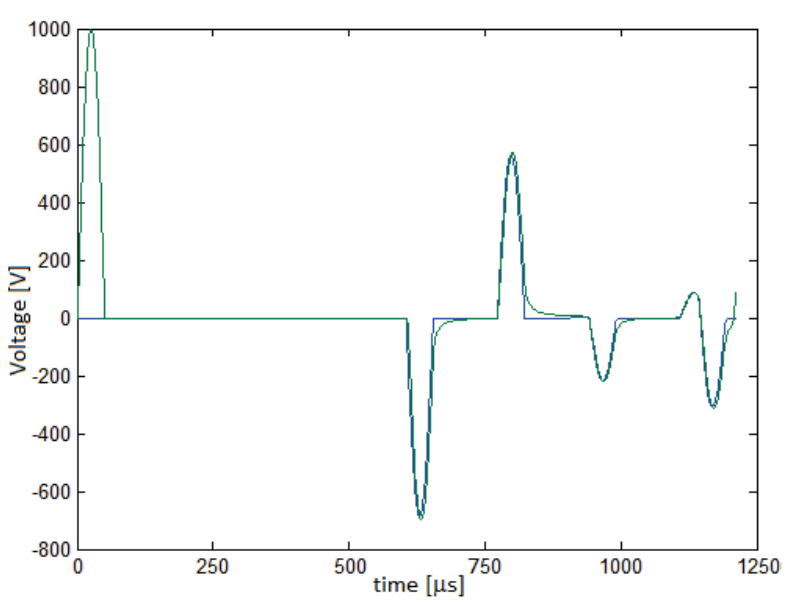

Figure 5 Reflected signal where $\mathrm{R}$ and $\mathrm{P}$ buses are disconnected



Figure 6 Reflected signal where $\mathrm{R}$ and $\mathrm{Z}$ buses are disconnected

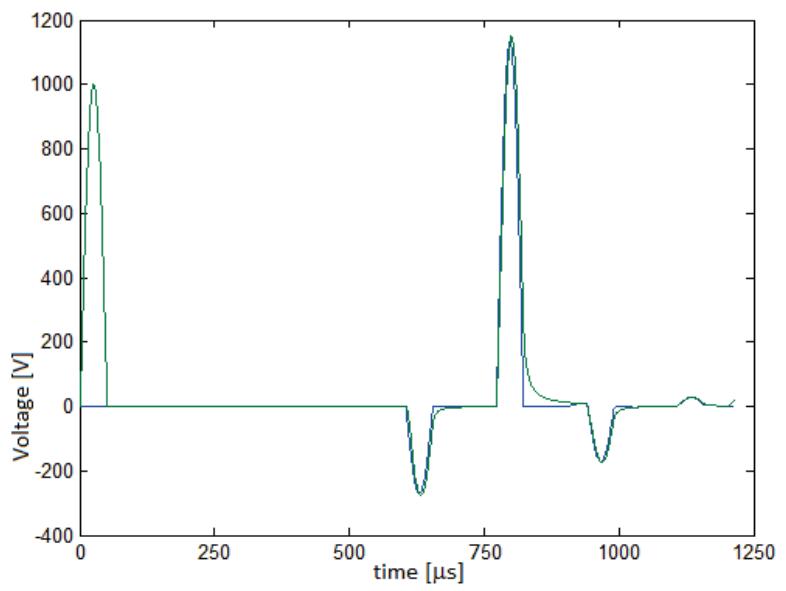

Figure 7 Reflected signal where TR and K bus are connected only

In all presented cases, control function is approximately equal to simulated signal. Amplitude of the expected reflected signals is significant enough that it is possible to presume that the reflection does exist and therefore line that is considered is connected. If there are multiple reflections from the same bus as is in the cases where the line S-K is involved, it is sufficient to observe only the first reflection since it often has the highest amplitude. If a certain reflected signal is superimposed with multiple reflected signal from other bus, for example in Fig. 2, their individual contribution is shown as value of vector $[a]$. In that case, from vector $[a]$, it is possible to determine which values are superimposed and if any value 
is significant enough it can be concluded that the transmission line is connected.

\section{CONCLUSION}

A new approach to topological processor using the travelling wave method is presented in this paper. Analysis results of simulated cases show that the presented theory works with the simple model of the test network and has potential for further development. Extra work is needed to use the proposed algorithm for large systems and meshed networks such as transmission network. Also, future work is required for optimization of locations of injection signal bus to make the entire network observable. Generally, the proposed method has a great potential in the future to improve and accelerate existing topological processors and improve operation of fast (PMU) state estimators.

\section{REFERENCES}

[1] Baseer, M. A. (2013). Travelling Waves for Finding the Fault Location in Transmission Lines. Journal Electrical and Electronic Engineering, 1(1), 1-19. https://doi.org/10.11648/j.jeee.20130101.11

[2] Korkali, M., Lev-Ari, H., \& Abur, A. (2012). TravellingWave-Based Fault-Location Technique for Transmission Grids Via Wide-Area Synchronized Voltage Measurements. IEEE Trans. on Power Syst., 27(2), 1003-1011. https://doi.org/10.1109/TPWRS.2011.2176351

[3] Marx, S., Johnson, B. K., Guzman, A., Skendzic, V., \& Mynam, M. V. (2013). Travelling Wave Fault Location in Protective Relays: Design, Testing and Results. $16^{\text {th }}$ Annual Georgia Tech Fault and Disturbance Analysis Conference, Atlanta, Georgia 6-7 May, 15 pp.

[4] Abur, A. \& Exposito, A. G. (2004) Power system state estimation: theory and implementation. Marcel Dekker Inc, New York. https://doi.org/10.1201/9780203913673

[5] Kansal, P. \& Bose, A. (2017). Bandwidth and Latency Requirements for Smart Transmission Grid Applications. IEEE Transactions on Smart Grid, 3(3), 1344-1352. https://doi.org/10.1109/TSG.2012.2197229

[6] Gol, M. \& Abur, A. (2014). A robust PMU based three-phase state estimator using modal decoupling. IEEE Trans Power Syst, 29(5), 2292-2299. https://doi.org/10.1109/TPWRS.2014.2299756

[7] Jones, K. D., Thorp, J. S., \& Gardner, R. M. (2013). Threephase linear state estimation using phasor measurements. In: Proceedings of the 2013 IEEE Power and Energy Society general meeting, Vancouver, 21-25 Jul, 5 pp. https://doi.org/10.1109/PESMG.2013.6672516

[8] Zhou, M., Centeno, V. A., Thorp, J. S., \& Phadke, A. G. (2006). An alternative for including phasor measurements in state estimator. IEEE Trans. Power Syst., 21(4), 1930-1937. https://doi.org/10.1109/TPWRS.2006.881112

[9] Chen, H., Zhang, L., Mo, J. et al. (2016). Synchrophasorbased real-time state estimation and situational awareness system for power system operation. J. Mod. Power Syst. Clean Energy, 4(3), 370-382. https://doi.org/10.1007/s40565-016-0212-9

[10] Ožegović, M. \& Ožegović, K. (1996). Electric Power Networks I. Udžbenici Sveučilišta u Splitu, Opal Computing d.o.o.

[11] van der Sluis, L. (2001). Transients in Power Systems, John Wiley\&Sons Ltd, Chichester. https://doi.org/10.1002/0470846186

[12] Chakrabarti, A. (2013). Power System Dynamics and Simulation. PHI Learning Private Limited, Delhi.
[13] Stojkov, M., Softić, A., \& Atić, M. (2015). Optimization of switching conditions in distribution power subsystem. Tehnički vjesnik, 22(5), 1297-1303. https://doi.org/10.17559/TV-20141211120022

\section{Contact information:}

Roko JERČIĆ, M. Eng.

Croatian Transmission System Operator, Ltd

Kupska 4, 10000 Zagreb, Croatia

E-mail: roko.jercic@hops.hr

Ivica PAVIĆ, PhD, Prof

University of Zagreb,

Faculty of Electrical Engineering and Computing,

Unska 3, 10000 Zagreb, Croatia

E-mail: ivica.pavic@fer.hr

Domagoj PEHARDA, PhD

Končar - Power Plant and Electric Traction Engineering, Inc. Fallerovo šetalište 22, 10000 Zagreb, Croatia

E-mail: domagoj.peharda@koncar-ket.hr 